\title{
IntelliPatent: a web-based intelligent system for fast chemical patent claim drafting
}

\author{
Pei-Hua Wang ${ }^{1}$ and Yufeng Jane Tseng ${ }^{1,2^{*}}$ (D)
}

\begin{abstract}
The first step of automating composition patent drafting is to draft the claims around a Markush structure with substituents. Currently, this process depends heavily on experienced attorneys or patent agents, and few tools are available. Intellipatent was created to accelerate this process. Users can simply upload a series of analogs of interest, and IntelliPatent will automatically extract the general structural scaffold and generate the patent claim text. The program can also extend the patent claim by adding commonly seen R groups from historical lists of the top 30 selling drugs in the US for all R substituents. The program takes MDL SD file formats as inputs, and the invariable core structure and variable substructures will be identified as the initial scaffold and R groups in the output Markush structure. The results can be downloaded in MS Word format (.docx). The suggested claims can be quickly generated with IntelliPatent. This web-based tool is freely accessible at https://intellipatent.cmdm.tw/.
\end{abstract}

Keywords: Web server, Markush structure, Pharmaceutical patent

\section{Introduction}

Claims are the most important sections in composition patents [1]. In the pharmaceutical industry, claims should include key compounds and all structural derivatives that are likely to have the same effects [2]. This is achieved by Markush structures [3] that describe a series of chemical compounds having a common core structure and variable substituents called R groups [4].

To ensure the novelty of a new composition patent, one needs to make sure that no compound in the claims is taken by prior arts. One common routine is to perform structure searches in chemical databases [5]. One of the most useful free online databases for chemical prior-art search is SureChEMBL [6]. Performing a structure search using the Markush structure as input is the most efficient method. To generate the input Markush structure, one needs to study the compounds they want to patent to identify the input scaffold and $\mathrm{R}$ group variations.

Previous studies focused on interpretation or searching of Markush structure. For an example, MarVis is capable

\footnotetext{
*Correspondence: yjtseng@csie.ntu.edu.tw

${ }^{1}$ Graduate Institute of Biomedical Electronics and Bioinformatics, National

Taiwan University, No. 1 Sec. 4, Roosevelt Road, Taipei 106, Taiwan

Full list of author information is available at the end of the article
}

of visualizing and analyzing Markush structures from a composition patent [7]. Its web based application, iMarVis, revised the underlying $R$ group numbering system to deal with nested $\mathrm{R}$ group presentation [8]. An algorithm based on SMIRK language is introduced to solve the query for a compound within a Markush structure [9].

The next step in drafting compositional patent claims is to maximize patent coverage. The algorithm implemented in ChemAxon can automatically generate Markush structures [10]. Periscope system helps generating Markush structures from compounds, visualization, and searching specific chemical structures [11]. However, both systems cannot expand the coverage by adding new variations to the Markush structure. If the patent coverage is fully maximized, a monopoly state of first-in-class drugs can be achieved and profits will be ensured [12,13].

Adding variations beyond the current Markush structure relies on the experience of patent attorneys. Hence, patent coverage becomes more or less dependent on the writers. Despite the advance in chemical informatics, drafting and describing Markush structures still requires substantial manual effort. In this study, we introduce a publicly available server, IntelliPatent, for rapid chemical 
patent drafting that includes the option to recommend Markush structures to expand composition patent claims. With an R group library built from 30 composition patents, the definition of output Markush structure can be extended based on the input compounds.

\section{Implementation \\ Dataset}

A library containing $269 \mathrm{R}$ groups was constructed from 30 patents of the top-selling drugs in the US in 2005. The compounds in the "Biological Study" or "Preparation" sections were retrieved from SciFinder with the patent IDs listed in the study by Hattori et al. [14]. The structures of the 30 drug entities were downloaded from DrugBank [15] using the trade names.

\section{Building the $\mathrm{R}$ group library}

The scaffolds of the 30 drug entities were identified using Scaffold Hunter-2.4.1 [16]. Using JChem Base API [17], the scaffolds of the compounds were removed to obtain 5589 structural fragments, which were saved as R groups. We removed 5183 duplicates including optical isomers and filtered out $2 \mathrm{R}$ groups having deuterium, 2 having phosphorus and 133 having aliphatic carbon chains with lengths longer than 6 atoms to have the final $269 \mathrm{R}$ groups.

Next, we identified 28 common groups along with their text terms from the patent claims. The common groups include functional groups with defined structures (e.g., hydroxyl, cyano and halo) and general groups that incorporate a series of structures (e.g., alkyl, aryl, and heteroaryl). The SMARTS notations for detecting the common groups were generated to categorize the $\mathrm{R}$ groups in the library into 57 categories, including composite categories such as alkyl-aryl. The common groups, which are defined as the substituents on carbon chains or rings, were also recorded.

\section{R groups from approved drugs}

The structures of 2413 approved drugs were retrieved from DrugBank on 2019/11/19. For those structures with more than one molecules, only the compound with the highest molecular weights were retained. We filtered out big molecules $(\mathrm{MW}>800 \mathrm{Da})$, tiny molecules $(\mathrm{MW}<100 \mathrm{Da}$ and molecules with less than 6 carbon atoms) and duplicates including optical isomers to have 1921 drugs. The R groups from 1808 drugs were obtained by removing the scaffolds from compounds as the methods we used in building $\mathrm{R}$ group library in this work. There are 364 different $\mathrm{R}$ groups from approved drugs.

\section{Expanding the coverage of the Markush structure}

The scaffold of the initial Markush structure, including the scaffold and collections of $\mathrm{R}$ groups attached to the same atoms, is generated by RDKit using the Find$M C S$ function from the input compounds. For every $\mathrm{R}$ group structure, the presence of common $\mathrm{R}$ groups will be detected. If the $\mathrm{R}$ group comprises several common groups, the text term describing the $\mathrm{R}$ group will be generated from the most distant common group moving to the ones near the attachment point. For $\mathrm{R}$ groups attached to a carbon chain or ring in the scaffold, if the $\mathrm{R}$ group structure exactly matches any of the common substituents on the corresponding main structure in our library, the rest of the common substituents will be added to the same ring or carbon chain.

\section{Case study data}

To demonstrate how IntelliPatent works, the chemical composition patents of bromazine, orphenadrine, omeprazole and timoprazole were retrieved from the European Patent Office using patent IDs US2527963A, US2567351A, EP0005129B1 and US4045563A, respectively. The example compounds for bromazine and omeprazole and the Markush structures of the subsets from the claims of orphenadrine and timoprazole were constructed manually.

\section{User's privacy}

The input file from user is deleted immediately after computation at the end of the session. Linux crontab command was used to check all the output files twice a day and remove the files that were created more than $12 \mathrm{~h}$ ago. Therefore, the output data including graph, text and MS Word files will be deleted in less than a day. Since the server does not generate or retain any log file, the IP address of users will not be traceable via the server of IntelliPatent. All web traffic is encrypted via HTTPS.

\section{Results and discussion $R$ group library analysis}

$269 \mathrm{R}$ groups are generated using the example compounds from the patents of 30 top-selling drugs and their scaffolds were processed by Scaffold Hunter, based on the concept that compounds in a Markush structure can be divided into a scaffold plus several $R$ groups.

The top $10 \mathrm{R}$ groups and their percentage of counts in all the $218 \mathrm{R}$ groups showing up at least twice among all 269 groups are shown in Table 1. The top duplicate $\mathrm{R}$ group, methyl, alone accounts for nearly $20 \%$ of occurrence. The top 10 groups account for $63 \%$ among all the duplicate $\mathrm{R}$ groups and they are all small groups 
Table 1 The top $10 \mathrm{R}$ groups in library

\begin{tabular}{lll}
\hline R group & SMILES & Occurrence (\%) \\
\hline Methyl & ${ }^{*} \mathrm{C}$ & 19.9 \\
Fluoro & ${ }^{*} \mathrm{~F}$ & 8.4 \\
Methoxy & ${ }^{*} \mathrm{OC}$ & 7.0 \\
Hydroxy & ${ }^{*} \mathrm{O}$ & 6.3 \\
Sulfamoyl & ${ }^{*} \mathrm{~S}(=\mathrm{O})(=\mathrm{O}) \mathrm{N}$ & 5.6 \\
Chloro & ${ }^{*} \mathrm{Cl}$ & 5.1 \\
Hydrogen & ${ }^{*} \mathrm{H}$ & 3.8 \\
Ethynyl & ${ }^{*} \mathrm{C} \mathrm{C}$ & 2.8 \\
Trifluoromethyl & ${ }^{*} \mathrm{C}(\mathrm{F})(\mathrm{F}) \mathrm{F}$ & 2.2 \\
Carboxy & ${ }^{*} \mathrm{C}(=\mathrm{O}) \mathrm{O}$ & 1.9 \\
\hline
\end{tabular}

Table 2 The top 10 most used $R$ groups from approved drugs

\begin{tabular}{llll}
\hline R group & SMILES & Occurrence (\%) & Both top 10 \\
\hline Methyl & ${ }^{*} \mathrm{C}$ & 23.6 & $\mathrm{O}$ \\
Hydroxy & ${ }^{*} \mathrm{O}$ & 18.2 & $\mathrm{O}$ \\
Chloro & ${ }^{*} \mathrm{Cl}$ & 6.4 & $\mathrm{O}$ \\
Amino & ${ }^{*} \mathrm{~N}$ & 5.1 & \\
Methoxy & ${ }^{*} \mathrm{OC}$ & 5.0 & 0 \\
Carboxy & ${ }^{*} \mathrm{C}(=\mathrm{O}) \mathrm{O}$ & 3.9 & $\mathrm{O}$ \\
Fluoro & ${ }^{*} \mathrm{~F}$ & 2.9 & $\mathrm{O}$ \\
Ethyl & ${ }^{*} \mathrm{CC}$ & 2.2 & \\
Hydroxymethyl & ${ }^{*} \mathrm{CO}$ & 1.7 & \\
Trifulromethyl & ${ }^{*} \mathrm{C}(\mathrm{F})(\mathrm{F}) \mathrm{F}$ & 1.0 & $\mathrm{O}$ \\
\hline
\end{tabular}

\section{Bromazine}

1. Compounds of the invention are those of the genral formula I

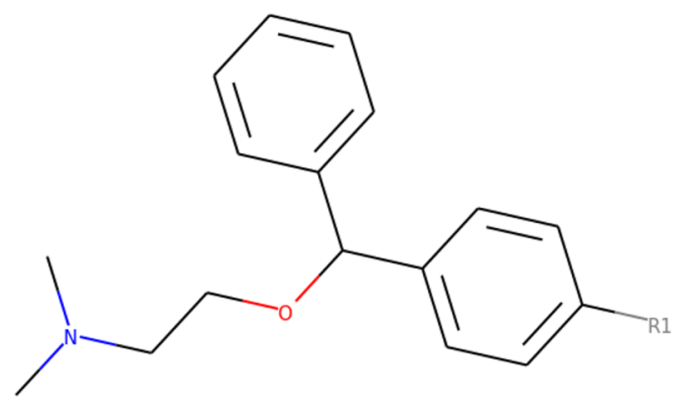

or a therapeutically acceptable salt thereof in which

$\mathrm{R} 1$ is a halogen atom, an alkoxy group, an alkyl group or a hydroxy group,

$\mathrm{R} 1$ can be at any position on the ring.

Fig. 2 The output claims using the four example compounds from the patent of bromazine (US2527963A)

containing less than 5 atoms. These 10 top duplicate $\mathrm{R}$ groups can be considered basic sets in $R$ group variations when drafting patent claims.

To see how much those R groups from top 30 best selling drugs overlapped with the DrugBank records, we extracted and analyzed the R groups of 1808 approved<smiles>CN(C)CCOC(c1ccccc1)c1ccc(F)cc1</smiles><smiles>CN(C)CCOC(c1ccccc1)c1ccc(Cl)cc1</smiles><smiles>CN(C)CCOC(c1ccccc1)c1ccc(Br)cc1</smiles><smiles>CN(C)CCOC(c1ccccc1)c1ccc(I)cc1</smiles>

Fig. 1 The chemical structures of compounds generated from the composition patent of bromazine (US2527963A) for demonstration 
<smiles>Cc1ccccc1C(OCCN(C)C)c1ccccc1</smiles>

Fig. 3 The active moieties of orphenadrine

drugs from DrugBank. There are 364 different $R$ groups from approved drugs. After comparison with our $\mathrm{R}$ group library, we found that $84.8 \%$ of the R groups from approved drugs are covered by our library. The top $10 \mathrm{R}$ groups with the highest occurrence from approved drugs is shown in Table 2.

There are 7 groups which appear in both of the top 10 $\mathrm{R}$ lists. This demonstrates that our $\mathrm{R}$ group library cover most of the $\mathrm{R}$ groups from approved small molecular drugs.

\section{Case study 1: bromazine}

We used bromazine, a first-generation antihistamine, to demonstrate how IntelliPatent generates patent claims for a series of input compounds. Bromazine was patented in 1950, and the composition patent contained only four compounds (see Fig. 1). The resulting claims contained a scaffold automatically generated from all the input compounds and several additional $\mathrm{R}$ groups and position variations with the corresponding text (Fig. 2).

This output shows another benefit and potential use for IntelliPatent, namely, broader coverage of the patent. The output claims from IntelliPatent using the bromazine compounds successfully covered the active moieties of orphenadrine (Fig. 3), an antihistamine of the same class patented in 1951, one year after the patent of bromazine. The four sets of compounds claimed in the patent of orphenadrine that are also covered by the output claims are shown in Fig. 4. Hence, IntelliPatent is capable of generating Markush structures for a series of analogs and expanding patent coverage by adding appropriate variations. The output claims can serve as a checklist to assist patent attorneys in designing claims.

\section{Case study 2: omeprazole}

The second drug for demonstration is omeprazole, the first clinically used proton pump inhibitor [18]. Omeprazole was patented in 1979, and there are 30 example compounds in the patent (see Additional file 1 for the structures). We use the 30 example compounds as input for IntelliPatent and the generated patent claims (see<smiles>CCOc1ccc(C(OCCN(C)C)c2ccccc2)cc1</smiles> 


\section{Omeprazole}

1. Compounds of the invention are those of the genral formula I

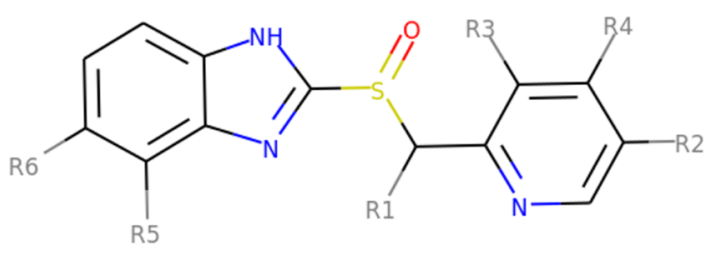

or a therapeutically acceptable salt thereof in which

$\mathrm{R} 1$ is a hydrogen atom or an alkyl group,

$\mathrm{R} 2$ is a halogen atom, an alkoxy group, an alkoxyalkoxy group, an alkyl group or a hydroxy group,

$\mathrm{R} 3$ is a halogen atom, a hydrogen atom, an alkoxy group, an alkyl group or a hydroxy group,

R4 is a halogen atom, a hydrogen atom, an alkoxy group, an alkyl group or a hydroxy group,

R5 is a halogen atom, a hydrogen atom, an alkanoyl group, an

alkoxy group, an alkoxycarbonyl group, an alkyl group or a hydroxy group, and

R6 is a halogen atom, a hydrogen atom, an alkanoyl group, an alkoxy group, an alkoxycarbonyl group, an alkyl group or a hydroxy group,

R2, R3, and R4 can be at any position on the ring,

$\mathrm{R} 5$, and $\mathrm{R} 6$ can be at any position on the ring.

Fig. 5 The output claims using the 30 example compounds from the patent of omeprazole (EP0005129B1)

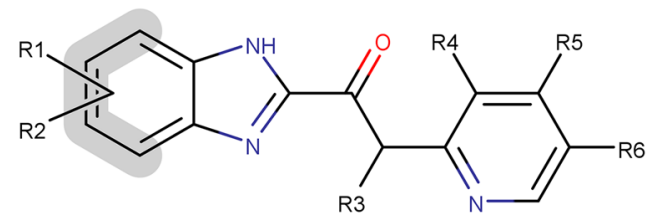

R1 and R2 are the same or different and are each halogen atom, hydrogen atom, alkoxy group, alkanoyl group, alkyl group,

hydroxy group

$\mathrm{R} 3$ is a hydrogen atom or a C1-3 alkyl group

R4, R5 and R6 are the same or different and are each halogen atom or C1-3 alkyl group

Fig. 6 The claims from timoprazole that were covered by the output using example compounds from omeprazole. The original patent claims of omeprazole (EP0005129B1) did not include the structures shown in the figure. The input structures to IntelliPatent are the example compounds in the patent of omeprazole. The two critical $\mathrm{R}$ groups that were absent in the original patent but present in our resulting claims are highlighted in red
Fig. 5) covered all the compounds claimed in the patent of omeprazole.

Our output claims include part of the claims from timoprazole, a proton pump inhibitor of the same class patented in 1977. The compounds from the patent of timoprazole that were covered by our generated claims, but not by those from omeprazole, are shown in Fig. 6 . The two critical $\mathrm{R}$ groups that were absent in the original patent but present in our resulting claims are the hydroxy group on the benzimidazole and halogen substituents on pyridine; the two critical $\mathrm{R}$ groups are highlighted in red in Fig. 6. If IntelliPatent were used in patent drafting of omeprazole, the patent coverage would be properly extended and these compounds could not be claimed by future patents. This implies that IntelliPatent is also useful after the introduction of Markush structure in pharmaceutical composition patents.

\section{Web server}

IntelliPatent is simple and intuitive to use, and users only need to upload and submit their compounds to use this service. Input file is accepted as MDL SD file, and the file should contain more than one compound that shares the same core structure. The output will be the patent claims with Markush structures and the $\mathrm{R}$ group definition text. The $R$ group variations will be broadened with the builtin $\mathrm{R}$ group library. Figure 7 shows the results page generated using the compounds in the composition patent of bromazine in Fig. 1 as input. The chemical scheme of the Markush structure is shown after the first sentence of the claims, and the definition of the R groups follows. The results can be downloaded in MS format by clicking the hypertext at the top of the result page.

\section{Conclusions}

IntelliPatent is a freely accessible web server that can automatically generate patent claims from input compounds and further expand claim coverage using an $R$ group library extracted from 30 patents. The resulting claim can serve as a first draft to reduce the human labor required to determine a suitable Markush structure from dozens or even hundreds of compounds. The results can also be used as a checklist while drafting claims to avoid omitting common variations in $\mathrm{R}$ groups that should be covered and protected. We hope that IntelliPatent can mitigate the heavy workload of patent attorneys and give scientists some insights into their compounds and the corresponding claims.

\section{Availability and requirements}

Project name: IntelliPatent.

Project home page: https://intellipatent.cmdm.tw/.

Operating system(s): Platform independent. 


\section{NATIONAL}

tAINAN

UNIVERSTIY

\section{IntelliPatent}

\section{Project Features \\ $\gg \underline{\text { Overview }}$}

IntelliPatent
» Patent Claim Generation
» $\underline{\text { Help }}$

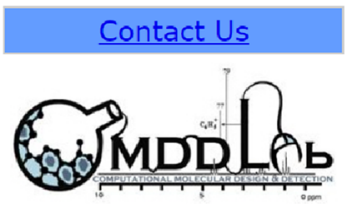

\section{Bromazine}

1. Compounds of the invention are those of the genral formula I

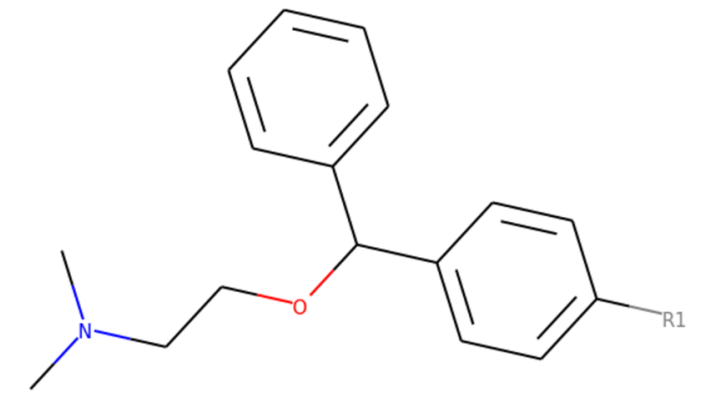

or a therapeutically acceptable salt thereof in which

$\mathrm{R} 1$ is a halogen atom, an alkoxy group, an alkyl group or a hydroxy group,

R1 can be at any position on the ring.

Copyright (C) 2018 Computational Molecular Design \& Metabolomics Laboratory. BEBI | CSIE | NTU |

Fig. 7 The result page of Intellipatent using the four example compounds from the patent of bromazine

Programming language: Python.

Other requirements: None.

License: None.

Any restrictions to use by non-academics: None.

\section{Supplementary information}

Supplementary information accompanies this paper at https://doi. org/10.1186/s13321-019-0401-4.

Additional file 1. Example compounds in the composition patent of omeprazole. The file contains all the structures of 30 example compounds from the patent of omeprazole (EP0005129B1) in MOL SD file format. This file was used as input in the case study of omeprazole.

\section{Acknowledgements}

Resources of the Laboratory of Computational Molecular Design and Metabolomics and the Department of Computer Science and Information Engineering of National Taiwan University were used in performing these studies.

\section{Authors' contributions}

PHW performed the analysis, implemented the server and drafted the manuscript. YJT conceived and supervised the study and edited the manuscript. Both authors read and approved the final manuscript.

\section{Funding}

This work was funded by the Ministry of Science and Technology of Taiwan under grant numbers MOST 107-2627-E-002-002, and National Taiwan University under grant numbers NTU-CC-108L893804.

\section{Availability of data and materials}

The tool is freely available at https://intellipatent.cmdm.tw/.

\section{Competing interests}

The authors declare that they have no competing interests.

\section{Author details}

${ }^{1}$ Graduate Institute of Biomedical Electronics and Bioinformatics, National Taiwan University, No. 1 Sec. 4, Roosevelt Road, Taipei 106, Taiwan. ${ }^{2}$ Department of Computer Science and Information Engineering, National Taiwan University, No. 1 Sec. 4, Roosevelt Road, Taipei 106, Taiwan. 
Received: 19 October 2019 Accepted: 2 December 2019

Published online: 11 December 2019

\section{References}

1. Shinmori A, Okumura M, Marukawa Y et al (2003) Patent claim processing for readability: structure analysis and term explanation. In: Proceedings of the ACL-2003 workshop on Patent corpus processing. 20:56-60. https:// doi.org/10.3115/1119303.1119310

2. Webber PM (2003) Protecting your inventions: the patent system. Nat Rev Drug Discov. 2:823-830. https://doi.org/10.1038/nrd1200

3. Valance EH (1961) Understanding the Markush claim in chemical patents. J Chem Doc. 1:87-92. https://doi.org/10.1021/c160002a022

4. Downs GM, Barnard JM (2011) Chemical patent information systems. WIREs Comput Mol Sci. 1:727-741. https://doi.org/10.1002/wcms.41

5. Simmons ES (1998) Prior art searching in the preparation of pharmaceutical patent applications. Drug Discov Today 3:52-60. https://doi. org/10.1016/S1359-6446(97)01145-8

6. Papadatos G, Davies M, Dedman N et al (2016) SureChEMBL: a large-scale, chemically annotated patent document database. Nucleic Acids Res 44:D1220-D1228. https://doi.org/10.1093/nar/gkv1253

7. Deng W, Berthel SJ, So WV (2011) Intuitive patent Markush structure visualization tool for medicinal chemists. J Chem Inf Model 51:511-520. https://doi.org/10.1021/ci100261u

8. Deng W, Scott E, Berthel SJ et al (2012) Deconvoluting complex patent Markush structures: a novel R-group numbering system. World Pat Inf 34:128-133. https://doi.org/10.1016/j.wpi.2012.02.002

9. Deng W, Schneider G, So WV (2011) Mapping chemical structures to Markush structures using SMIRKS. Mol Inform 30:665-671. https://doi. org/10.1002/minf.201100041
10. Kovács P, Botka G, Figyelmesi Á (2019) Automatic generation of Markush structures from specific compounds. World Pat Inf 57:59-69. https://doi. org/10.1016/j.wpi.2019.03.006

11. Cosgrove DA, Green KM, Leach AG et al (2012) A system for encoding and searching Markush structures. J Chem Inf Model 52:1936-1947. https:// doi.org/10.1021/ci3000387

12. Van DijkT (1996) Patent height and competition in product improvements. J Ind Econ 44:151-167. https://doi.org/10.2307/2950643

13. Kitch EW (2000) Elementary and persistent errors in the economic analysis of intellectual property. Vand L Rev 53:1727

14. Hattori K, Wakabayashi H, Tamaki K (2008) Predicting key example compounds in competitors' patent applications using structural information alone. J Chem Inf Model 48:135-142. https://doi.org/10.1021/ci7002686

15. Wishart DS, Knox C, Guo AC et al (2006) DrugBank: a comprehensive resource for in silico drug discovery and exploration. Nucleic Acids Res 34:D668-D672. https://doi.org/10.1093/nar/gkj067

16. Wetzel S, Klein K, Renner S et al (2009) Interactive exploration of chemical space with Scaffold Hunter. Nat Chem Biol 5:581-583. https://doi. org/10.1038/nchembio.187

17. ChemAxon (2015) JChem. Release 15.5.4. https://www.chemaxon.com

18. Strand DS, Kim D, Peura DA (2017) 25 years of proton pump inhibitors: a comprehensive review. Gut Liver 11:27-37. https://doi.org/10.5009/gnl15 502

\section{Publisher's Note}

Springer Nature remains neutral with regard to jurisdictional claims in published maps and institutional affiliations.
Ready to submit your research? Choose BMC and benefit from:

- fast, convenient online submission

- thorough peer review by experienced researchers in your field

- rapid publication on acceptance

- support for research data, including large and complex data types

- gold Open Access which fosters wider collaboration and increased citations

- maximum visibility for your research: over $100 \mathrm{M}$ website views per year

At BMC, research is always in progress.

Learn more biomedcentral.com/submissions 